\title{
Hemodynamic Parameters Predict the In-Stent Thrombosis After the Multibranched Endovascular Repair for Complex Abdominal Aortic Aneurysms: A Preliminary Study on Branched Stent-Graft Thrombosis.
}

Ming-Yuan Liu

Beijing Friendship Hospital

Yunqing Mu

Beihang University

Yang Jiao

Peking University People's Hospital

Junjun Liu

Affiliated Hospital of Qingdao University

Simeng Zhang

Fu Wai Hospital

Xiaoyan Deng

Beihang University

Wei Li

Peking University People's Hospital

Anqiang Sun ( $\nabla$ anqiangsun@163.com )

Beihang University

\section{Research Article}

Keywords: In-Stent Thrombosis (IST), Branched Stent-Grafts (BSG), Computational Fluid Dynamic, Endovascular Aortic Repair (EVAR), Biomechanics.

Posted Date: December 31st, 2020

DOI: https://doi.org/10.21203/rs.3.rs-133348/v1

License: (c) (i) This work is licensed under a Creative Commons Attribution 4.0 International License.

Read Full License 
1

2 Title: Hemodynamic Parameters Predict the In-stent Thrombosis

3 after the Multibranched Endovascular Repair for Complex

4 Abdominal Aortic Aneurysms: A Preliminary Study on Branched

5 Stent-graft Thrombosis.

6

7 Running head: Hemodynamic Index for In-Stent Thrombosis

8

9 Original article

10

Abstract: 198 words

Word Count: 2555 words

\section{Authors:}

Ming-Yuan $\mathrm{Liu}^{1 *} \mathrm{MD}, \mathrm{PhD}$, Yunqing $\mathrm{Mu}^{2,3 *}$, Yang Jiao ${ }^{4} \mathrm{MD}, \mathrm{PhD}$, Junjun $\mathrm{Liu}^{5} \mathrm{MD}$, $\mathrm{PhD}$, Simeng Zhang ${ }^{6,7} \mathrm{MD}, \mathrm{PhD}$, Xiaoyan Deng ${ }^{2,3}, \mathrm{PhD}$, Wei $\mathrm{Li}^{4+4} \mathrm{MD}, \mathrm{PhD}$ and Anqiang $\operatorname{Sun}^{2,3 \#}, \mathrm{PhD}$

${ }^{1}$ Department of Vascular Surgery, Beijing Friendship Hospital, Capital Medical University, Beijing 100050, China

${ }^{2}$ School of Biological Science and Medical Engineering, Beihang University, Beijing, 100083, China

${ }^{3}$ Beijing Advanced Innovation Center for Biomedical Engineering, Beihang University, Beijing, 100083, China

${ }^{4}$ Department of Vascular Surgery, Peking University People's Hospital, Beijing 100044, China

${ }^{5}$ Department of Vascular Surgery, The Affiliated Hospital of Qingdao University, 
Qingdao 266003, China

${ }^{6}$ Department of Vascular Surgery, Changhai Hospital, Shanghai, China

${ }^{7}$ Department of Pediatric Cardiac Surgery, State Key Laboratory of Cardiovascular Disease, Fuwai Hospital, National Center for Cardiovascular Disease, Chinese Academy of Medical Sciences and Peking Union Medical College, Beijing, China

*These authors contributed equally to this work.

Authors information:

Ming-Yuan Liu MD, PhD, Email: dr.mingyuanliu@pku.edu.cn

Yunqing Mu, Email: qymoment@126.com

Yang Jiao MD, PhD, Email: drversatile@ 126.com

Junjun Liu MD, PhD, Email: surgicalmaster@163.com

Simeng Zhang MD, PhD, Email: dr_dreamdealer@ 163.com

Xiaoyan Deng, PhD, Email: dr_newbrain@163.com

Wei Li MD, PhD, Email: mailtowei76@qq.com.

Anqiang Sun, PhD, Email: anqiangsun@163.com

\section{\#Corresponding authors:}

Anqiang Sun, School of Biological Science and Medical Engineering, Beihang University, Beijing 100083, China. Email: angiangsun@163.com or Wei Li, Department of Vascular Surgery, Peking University People's Hospital, Beijing 100044, China. Email: mailtowei76@qq.com.

\section{Ethics approval and consent to participate}

The authors confirmed that all the experiment protocol for involving human data was in accordance to guidelines of national/international/institutional or Declaration of Helsinki in the manuscript. Informed Consent were obtained from all the patients and the study protocols were approved by the Ethical Review Board and Statistics 
Department of Peking University People's Hospital (No. 2017 PHB166-01).

\section{Consent for publication}

All the consents of the participants were obtained for publication.

\section{Abstract}

Backgrounds: Branched vessel occlusion is frequently reported in the endovascular repair for aortic pathology. This study aimed to evaluate the hemodynamic indicators associated with the in-stent thrombosis (IST) of the branched stent-graft (BSG) after the endovascular aortic repair (EVAR) for complex abdominal aortic aneurysm.

Methods: A retrospective evaluation was performed based on the computed tomography (CT) scan and clinical data of three patients who underwent multibranched endovascular repair. The reconstruction of patient-specific 3-dimension models and hemodynamic analysis for IST were performed. The hemodynamic related parameters including time-averaged wall shear stress (TAWSS), oscillatory shear stress index (OSI) and relative residence time (RRT) were compared between the individual data.

Results: The velocity, TAWSS, OSI and RRT were radically changed in the area of IST. The IST of the BSG were located at the hemodynamic alteration regions near the bending curve where the decreased flow velocity $(<0.6 \mathrm{~m} / \mathrm{s})$, TAWSS $(<0.8 \mathrm{~Pa})$ and elevated OSI ( $>0.3)$, and RRT ( $>5$ s) were constantly observed.

Conclusions: The hemodynamic perturbations in the BSG predispose the IST, which could be predicted by a series changes of the flow parameters. Early hemodynamic analysis might be useful for identifying and remediating the IST after the multibranched 
endovascular repair.

79 Trial registration: This study was retrospectively registered and approved by the 80 institutional ethical review board.

81 Keywords: In-Stent Thrombosis (IST), Branched Stent-Grafts (BSG), Computational Fluid Dynamic, Endovascular Aortic Repair (EVAR), Biomechanics.

\section{List of abbreviations}

AAA - Abdominal Aortic Aneurysm

EVAR - Endovascular Aortic Repair

BSG - Branched Stent-Grafts

IST - In-Stent Thrombosis

CT - Computed Tomography

90

CA - Celiac Artery

SMA - Superior Mesenteric Artery

LRA - Left Renal Artery

RRA - Right Renal Artery

TAWSS - Time-Averaged Wall Shear Stress

OSI - Oscillatory Shear Stress Index

RRT - Relative Residence Time 
101

102

Abdominal aortic aneurysm (AAA) is a disease with a high incidence and mortality ${ }^{1}$ Endovascular aortic repair (EVAR) has been emerged as the "first choice" of the minimally invasive technique to treat aortic aneurysms ${ }^{2}$. For the patients with complex abdominal aortic aneurysms that were not anatomically amenable to standard $\mathrm{EVAR}^{3}$, a variety of approaches, such as the fenestrated stent-graft ${ }^{4}$, branched stent-graft ${ }^{5}$ and chimneys technique ${ }^{6}$ have been reported to the multibranched endovascular repair. However, the branched stent-grafts (BSG) occlusion caused by the in-stent thrombosis (IST) has been reported in the clinical researches ${ }^{7,8}$, often leading to organ malperfusion ${ }^{9}$. Morphological analysis alone might be insufficient to comprehensively evaluate the IST. Hemodynamics was considered to play a critical role in the formation and progression of in-stent restenosis ${ }^{10,11}$. Hemodynamic parameters might reveal the flow status of BSG and provide quantitative evaluations of the IST and thus inform medical decision-making. However, the mechanism and the hemodynamic characteristics of IST in the BSG were little known. Therefore, we aimed to evaluate the IST related hemodynamics features of the stent-graft in this study. Three cases that underwent BSG treatment were selected for this study. The models of the aorta and BSG were constructed based on the computed tomography (CT) images and the hemodynamic features were numerically simulated and investigated. We revealed the potential mechanism and identified the major hemodynamic factors for the IST of BSG. Furthermore, based on the analyzed results, some suggestions were presented to help 
121

122

123

124

125

126

127

optimizing the implantation of the current commercial-available stent-grafts and avoiding the latent pitfall.

\section{Methods}

\section{Models}

The CT images of three patients were imported into the Mimics software (v19.0, Materialise, Ann Arbor, MI, USA) to build three-dimensional (3D) models. All CT scan images were obtained from Peking University People's Hospital (Beijing, China). Informed Consent were obtained from all the patients and the study protocols were approved by the Ethical Review Board and the Statistics Department of Peking University People's Hospital (No. 2017 PHB166-01). The pre- and post-operative CT scan images of three cases are shown in the upper and lower lines of Fig. 1(a), respectively. All three patients with complex aortic aneurysms were treated with physician-modified BSG repair for the aorta (Endurant, Medtronic, Santa Rosa, Calif)) and the visceral arteries (Viabahn, Gore Inc). The procedures details are illustrated in Fig. 2. The celiac artery (CA), superior mesenteric artery (SMA), left renal artery (LRA), and right renal artery (RRA) were repaired in the Case 1, while the SMA and the bilateral renal arteries were repaired in both Case 2 and Case 3 . In order to unify the models for comparison between different cases, the celiac artery in case 1 model was removed. The models were smoothed and optimized in Geomagic Studio software (3D System, Morrisville, NC, USA) for meshing process. 
141

142

143

144

145

146

147

148

149

150

151

152

153

154

155

156

157

158

159

160

\section{Mesh generation}

Models were meshed using ICEM software (ANSYS, Inc., Canonsburg, PA, USA). To acquire the same degree of accuracy for all simulations, the same max global base cell size of $2 \mathrm{~mm}$ was used for each model. A hybrid meshing method ${ }^{12}$ that comprises both tetrahedral and hexahedral elements was used in all models. In addition, prism layers were created near the boundaries to ensure the accuracy of the model meshing. The total number of elements in models of case 1-3 is 993470,805931 and 785617 respectively. The meshes of three models are shown in Fig. 1(b).

\section{Boundary conditions}

The simulations were performed under pulsatile flow conditions, with the velocity profile (Fig. 1(c)) as inlet boundary conditions ${ }^{13-15}$. Outflow conditions have been used as outlet conditions, with the volume flow divided of $20.2 \%$ to the SMA, $19 \%$ to each renal artery, and $41.8 \%$ to the infrarenal aorta ${ }^{16,17}$. The flow ratio of each branch is marked in Fig. 1(b).

\section{Assumptions and governing equations}

The blood was assumed as incompressible Newtonian fluid ${ }^{18}$ with a dynamic viscosity of $0.0035 \mathrm{~kg} \cdot \mathrm{m}^{-1} \cdot \mathrm{s}^{-1}$ and a density of $1050 \mathrm{~kg} / \mathrm{m}^{3}$. The numerical simulation was based on the Navier-Stokes equation (neglecting the gravity) and the conservation of mass:

$$
\rho\left[\frac{\partial \vec{u}}{\partial t}+(u \cdot \nabla) \vec{u}\right]+\nabla p-\mu \nabla^{2} \vec{u}=0
$$

$$
\nabla \cdot \vec{u}=0
$$


161

162

Where $\vec{u}$ and $\mathrm{p}$ respectively represent the fluid velocity vector and the pressure. $\rho$ and $\mu$ are the density and viscosity of blood. The CFD software package, ANSYS Fluent 14.5 (ANSYS, Lebanon, NH, USA) was used for the simulations. The pressurebased solver and SIMPLE algorithm were used for calculation.

The convergence criterion was set to be $10^{-5}$ for both continuity and velocity residuals. A uniform time-step of $0.005 \mathrm{~s}$ was chosen for all simulations. For each model, four cardiac cycles were carried out in each simulation process to obtain a periodic solution ${ }^{19}$, and the results of the final cycle were presented for post-processing and analysis ${ }^{20}$.

\section{WSS-related parameters of interest}

This study took a particular close look on the various near-wall hemodynamic (NWH) parameters that have been shown to have an effect on thrombus formation. Therefore, the time-averaged wall shear stress (TAWSS) used to describe the general features of WSS in the pulsatile cycle was analysed. The TAWSS was defined as follows:

$$
T A W S S=\frac{1}{T} \int_{0}^{T}|W S S(s, t)| \cdot d t
$$

where $T$ is the duration of the cardiac cycle, WSS is the instantaneous wall shear stress vector, and $s$ is the position on the vessel (or stent-grafts) wall.

The oscillatory shear stress index (OSI) is a parameter which can describe the changing frequency of the WSS direction. It ranges from 0 , where the flow is one-directional without oscillations, to 0.5 , where the WSS direction frequently changes.

The OSI on the inner wall of the models was calculated as ${ }^{21,22}$ :

$$
O S I=0.5\left[1-\left(\frac{\left|\frac{1}{T} T_{0}^{T} W S S(s, t) \cdot d t\right|}{\frac{1}{T} \int_{0}^{T}|W S S(s, t)| \cdot d t}\right)\right]
$$


where WSS is a vector parameter and its direction changes with the cardiac cycle time. $T$ is the duration of the cardiac cycle and $s$ is the position on the vessel (or stent-grafts) wall.

The relative residence time (RRT) is generally used to characterize the length of time that near-wall particles stay at the wall ${ }^{23}$. It is a metric that can reflect both OSI and TAWSS $^{24}$. It is defined as:

$$
\mathrm{RRT}=\frac{1}{(1-2 \cdot O S I) \cdot T A W S S}
$$

\section{Results}

\section{Clinical outcome}

Table 1 shows the demographic characteristics and clinical outcome of the three patients. No complication was observed at the postoperative period of the three cases. During the follow-up, the Case 2 have shown the abdominal pain at the 6 months after the hospital discharge. The CT angiography revealed that there was thrombosis in the BSG of SMA and LRA (Fig. 3). But there were no IST detected in both Case 1 and Case 3 (Fig. 3).

\section{Flow velocity}

Figure 4 shows the velocity results of three cases at four continuous moments through the last cardiac cycle used four simulations $\left(\mathrm{t}_{1}=0.125 \mathrm{~s}, \mathrm{t}_{2}=0.225 \mathrm{~s}, \mathrm{t}_{3}=0.435 \mathrm{~s}, \mathrm{t}_{4}=0.62 \mathrm{~s}\right)$. To reveal the flow characteristics of the BSG, the parameters are illustrated on the branched arterial region. Streamlines in three cases are shown in Fig. 4(a-f), and the 
magnitude of velocity is indicated in color. The locations of thrombosis in Case 2 were precisely matched with the identified low-velocity areas in Fig. 4(c) where the IST of BSG were occurred. To investigate the velocity change in these low-velocity regions, the cross-sections were created in the model of Case 1, Case 2, and the Case 3. Contours of velocity in different sections were also shown (Fig. 4(b, d, f)). It was observed that the thrombosis/IST areas in Case 2, marked in black circle (Fig. 4 (c)), seemed to have lower velocity than the corresponding area of Case 1(Fig. 4 (a)) and Case 3 (Fig. 4 (e)), and even the upstream and downstream areas of itself.

\section{WSS, TAWSS, OSI and RRT}

Figure 5 have shown the WSS distributions of the BSG through four continuous moments in a cardiac cycle of the three cases. There was no remarkable change in the WSS along the BSG in the Case 1 and Case 3. Nevertheless, the low WSS regions appeared with passing time in a pulsation period were observed in Case 2 and the regions were matched with the IST area. Therefore, the hemodynamic parameters including TAWSS, OSI and RRT were analyzed to visualize the quantitative characteristics of the three cases. Contours of TAWSS, OSI and RRT on the wall of the stent-grafts are shown in Fig. $6(\mathrm{a}-\mathrm{c})$ respectively. We found the IST area were constantly matched with the regions that TAWSS, OSI and RRT were significantly altered in the BSG. 

comparison between groups were analyzed (Fig 7). The velocity $(\mathrm{m} / \mathrm{s})$ of the thrombosis/IST areas in Case $2(0.40 \pm 0.17)$ were significantly lower than the corresponding bending area of Case $1(1.08 \pm 0.23)(\mathrm{P}<0.01$, Fig. 7(a)) and Case $3(1.01$ $\pm 0.12)(\mathrm{P}<0.05$, Fig. $7(\mathrm{a}))$. The value of TAWSS $(\mathrm{Pa})$ in the IST areas $(0.65 \pm 0.06)$

227 was decreased $(\mathrm{P}<0.01$, Fig.7(b)) compared with the corresponding area in Case $1(0.93$ $228 \pm 0.09)$ and Case $3(0.88 \pm 0.06)$. However, in the IST areas, the value of OSI $(0.30 \pm$ $0.04)$ and RRT(s) $(9.27 \pm 2.61)$ were higher than the value in Case $1(0.06 \pm 0.03),(1.94$ $\pm 0.24)$ and in Case $3(0.11 \pm 0.06),(2.52 \pm 1.00)$, respectively $(\mathrm{P}<0.05$, Fig.7(c-d)).

231 Except the parameter of the IST area, the rest of the flow characteristics were consistent and there were no abnormal OSI, RRT and TAWSS observed. The IST area had the consistently lower values of velocity $(<0.6 \mathrm{~m} / \mathrm{s})$ and TAWSS $(<0.8 \mathrm{~Pa})$ and higher values of OSI $(>0.2)$ and RRT $(>5 \mathrm{~s})$ compared with the none thrombosis region in the BSG.

\section{Discussion}

Since BSG was introduced in the repair of complex aortic aneurysms ${ }^{5}$, studies $^{25-27}$ have amended the technique and demonstrated its feasibility and durability for the patients with complex aortic aneurysms. Even with high technical success, however, the visceral branch IST remain one of the leading complications for the BSG implantation ${ }^{28,29}$. The 
241

242

243

flow characteristics of the BSG and its relationship with the IST remain largely unknown. Computational fluid dynamics were performed in this study to evaluate the hemodynamic mechanism of thrombosis within the visceral branches and explore the IST related hemodynamic parameters.

It is known that low velocity was associated with the thrombosis. As shown in the velocity streamline diagram (Fig. 4), the BSG of Case 1 and Case 3 had a higher velocity and a uniform flow pattern than the velocity of Case 2 and the IST were observed in the marked areas (black circle). In case 2, the velocity in the three marked areas were significantly lower than the upstream and downstream areas of the BSG (Fig 4), and it was also lower than corresponding locations in other two cases (Fig 7).

Theoretically, low velocity induced low TAWSS distribution ${ }^{30}$. Our result demonstrated that the relative low TAWSS $(<0.8 \mathrm{~Pa})$ (Fig 5.) regions was matched with the regions of low velocity $(<0.6 \mathrm{~m} / \mathrm{s})$ (Fig 4.). Because the relevance of the WSS-related NWH hemodynamic parameters (Fig. 6), we compared the TAWSS, OSI (an index to evaluate the fluctuation of the blood flow) and RRT (relative residence time of the blood flow) and determined the consistence of these parameter in the tendency. It was reported that low WSS, high OSI and high RRT will promote platelet aggregation and finally lead to thrombus formation ${ }^{30-32}$. In our results, the flow characteristics including TAWSS, OSI, and the RRT were consistent in the none-thrombosis area whereas hemodynamic alteration was solely revealed in the IST area. By analyzing the distribution these parameters, we showed that the IST area (Fig.3) were matched with the low velocity area (Fig.4). Furthermore, it matched with the areas of reduced TAWSS (Fig. 6(a)), 
elevated OSI (Fig. 6(b)) and RRT (Fig. 6(c)). Together, these data have demonstrated that the hemodynamic perturbations introduced the radical changes of the hemodynamic parameters, which may predispose the IST. The IST was able to be predicted by the alteration of the hemodynamic parameters.

In clinical practice, longer length of BSGs than the original vessels were usually inevitably required for the convenience of cannulation in the treatment of complex aortic aneurysms. Although a longer BSG would provide more gradual changes in momentum and allow blood flow to develop before reaching the target vessel ${ }^{33}$, it would inevitably result in a curved portion of the BSG, where the blood flow would be disturbed and prone-thrombus environments would be created. In the present study, the three regions of IST after the procedure were developed at the bending region of the BSG, which suggested that a sharp curvature of the stent-grafts would adversely affect blood flow near the curved location, thereby accelerating thrombus formation. Therefore, preoperative planning to avoid the sharp curvature of the bending endografts was advocated.

Also, the diameter of the BSG at IST location were larger compared with the curvature section. The inconsistent size of the diameter may attribute to metal fatigue of the BSG. Metal fatigue and fracture have been reported to limit the efficacy of the stents and result in $\mathrm{IST}^{34}$. The discontinuous diameter of the BSG caused the varied velocity flow fields near the curved region. The variable flow field characterized by flow separation and stagnation with low fluid velocity and low WSS were related to thrombosis and stenoses $^{10,35,36}$. In particular, the curvature magnitude in morphology and the change 
in diameter near the curved region can both contribute to the hemodynamic changes. To date, researchers have illustrated the evolution from physician-modified to company-manufactured fenestrated-branched endografts in the treatment of complex and thoracoabdominal aortic aneurysms ${ }^{4,37}$. However, the BSGs were inevitably used in the multibranched endovascular aortic repair scenarios. Despite the refinement of the stent-grafts, preoperative designing the morphological configuration of the BSG should also be considered. Our results suggested that the sharp bending curve of the BSG should be avoided and early postoperative hemodynamic assessments of the BSG could assist in detecting the IST-predisposing after the operation. If significant changed hemodynamic regions are calculated in the BSG, special attention needs to be paid to these regions, as it may involve a higher risk of IST. Our results indicated that the relatively lower velocity $(<0.6 \mathrm{~m} / \mathrm{s})$, TAWSS $(<0.8 \mathrm{~Pa})$ and higher OSI $(>0.2)$ and RRT $(>5 \mathrm{~s})$ distribution were associated with the IST in the BSG. Therefore, the relatively higher OSI, RRT and lower TAWSS areas of the BSG may be the predictors of IST. Compared to previous ideal model used to study branched stent configurations ${ }^{17,33,38}$, the models used in this study were derived from clinical cases, which examined and added the current knowledge with "real-world" data. Moreover, the models in this study provided important insights on the velocity and the WSS distribution of the BSG.

However, several limitations in this study should be mentioned. First, only the fluid domain of the blood vessel is used for simulation. Although the interaction between stent-grafts and blood flow with the pulsatility of the flow were not considered in this study, the results still solid because only insignificant displacements were found in 
stent-grafts during the cardiac cycle and it was a common practice in the CFD analysis ${ }^{39}$. Second, the sample was limited. Only three patient-specific models were used for computation in our study due to the rarity of treatment. Further evaluation with large sample size, patient specific boundaries, and long-term follow-up will be suggested to further validate these findings.

\section{Conclusion}

This study identified that the aberrant hemodynamic parameters predispose the IST of in the BSG. Analysis of these parameters in the early postoperative period may be beneficial for identifying and remediating the IST after the multibranched endovascular aortic repair.

\section{Declarations}

\section{Ethics approval and consent to participate}

The authors confirmed that all the experiment protocol for involving human data was in accordance to guidelines of national/international/institutional or Declaration of Helsinki in the manuscript. Informed Consent were obtained from all the patients and the study protocols were approved by the Ethical Review Board and Statistics Department of Peking University People's Hospital (No. 2017 PHB166-01).

\section{Consent for publication}

All the consents of the participants were obtained for publication. 


\section{Availability of data and materials}

The data that support the findings of this study are available from Peking University People's Hospital but restrictions apply to the availability of these data, which were used under license for the current study, and so are not publicly available. Data are however available from the authors upon reasonable request and with permission of the Ethical Review Board, Peking University People's Hospital.

\section{Competing interests}

The author(s) declared no potential conflicts of interest with respect to the research, authorship, and/or publication of this article.

\section{Funding}

This work was supported by the National Natural Science Foundation of China (No. 11872096, 11472031, 82000429 and 81800403), Beijing Municipal Hospital Scientific Research Training Program Foundation (PX20210105) and Scientific Research Program of Beijing Education Commission (KM202110025016).

\section{Authors' contributions}

MY Liu, AQ Sun, W Li, and XY Deng were contributed to the conception and study design/obtaining funding/ writing the article/critical revision of the article. YQ Mu, Y Jiao, and JJ Liu were contributed to the data collection/analysis and interpretation. SM Zhang was contributed to the obtaining funding.

\section{Acknowledgements}

The authors appreciated the assistance from Statistics Department of Peking University People's Hospital. 


\section{Authors' information (optional)}

The ORCID of Dr. MY- Liu is https://orcid.org/0000-0002-6449-4885.

\section{References}

1. Morris L, Delassus P, Walsh M, McGloughlin T. A mathematical model to predict the in vivo pulsatile drag forces acting on bifurcated stent grafts used in endovascular treatment of abdominal aortic aneurysms (AAA). $J$ Biomech. 2004;37(7):1087-1095.

2. Li Z, Kleinstreuer C. Analysis of biomechanical factors affecting stent-graft migration in an abdominal aortic aneurysm model. $J$ Biomech. 2006;39(12):2264-2273.

3. West CA, Noel AA, Bower TC, Cherry KJ, Jr., Gloviczki P, Sullivan TM, et al. Factors affecting outcomes of open surgical repair of pararenal aortic aneurysms: a 10-year experience. J Vasc Surg. 2006;43(5):921-927; discussion 927-928.

4. Oderich GS, Ribeiro MS, Sandri GA, Tenorio ER, Hofer JM, Mendes BC, et al. Evolution from physician-modified to company-manufactured fenestratedbranched endografts to treat pararenal and thoracoabdominal aortic aneurysms. J Vasc Surg. 2019;70(1):31-42 e37.

5. Anderson J, Nykamp M, Danielson L, Remund T, Kelly PW. A novel endovascular debranching technique using physician-assembled endografts for repair of thoracoabdominal aneurysms. J Vasc Surg. 2014;60(5):1177-1184.

6. Tricarico R, He Y, Laquian L, Scali ST, Tran-Son-Tay R, Beck AW, et al. 

Following Chimney Endovascular Repair of Juxtarenal Aortic Aneurysms. $J$ Endovasc Ther. 2017;24(6):880-888.

7. Polanczyk A, Podyma M, Stefanczyk L, Szubert W, Zbicinski I. A 3D model of thrombus formation in a stent-graft after implantation in the abdominal aorta. $J$ Biomech. 2015;48(3):425-431.

8. Starnes BW. Physician-modified endovascular grafts for the treatment of elective, symptomatic, or ruptured juxtarenal aortic aneurysms. J Vasc Surg. 2012;56(3):601-607.

9. Ou J, Chan YC, Cheng SW. A Systematic Review of Fenestrated Endovascular Repair for Juxtarenal and Short-Neck Aortic Aneurysm: Evidence So Far. Ann Vasc Surg. 2015;29(8):1680-1688.

10. Wootton $\mathrm{DM}, \mathrm{Ku} \mathrm{DN}$. Fluid mechanics of vascular systems, diseases, and thrombosis. Annu Rev Biomed Eng. 1999;1:299-329.

11. Malek AM, Alper SL, Izumo S. Hemodynamic shear stress and its role in atherosclerosis. JAMA. 1999;282(21):2035-2042.

12. Chiastra C, Morlacchi S, Pereira S, Dubini G, Migliavacca F. Computational fluid dynamics of stented coronary bifurcations studied with a hybrid discretization method. European Journal of Mechanics B-Fluids. 2012;35:7684.

13. Aronberg DJ, Glazer HS, Madsen K, Sagel SS. Normal thoracic aortic diameters by computed tomography. J Comput Assist Tomogr. 1984;8(2):247-250. 
14. Moore JE, Jr., Ku DN. Pulsatile velocity measurements in a model of the human abdominal aorta under resting conditions. J Biomech Eng. 1994;116(3):337-346.

15. Taylor CA, Hughes TJ, Zarins CK. Finite element modeling of threedimensional pulsatile flow in the abdominal aorta: relevance to atherosclerosis. Ann Biomed Eng. 1998;26(6):975-987.

16. Ku DN, Glagov S, Moore JE, Jr., Zarins CK. Flow patterns in the abdominal aorta under simulated postprandial and exercise conditions: an experimental study. J Vasc Surg. 1989;9(2):309-316.

17. Suess T, Anderson J, Sherman A, Remund T, Pohlson K, Mani G, et al. Shear accumulation as a means for evaluating risk of thromboembolic events in novel endovascular stent graft designs. J Vasc Surg. 2017;65(6):1813-1819.

18. Wang ZZ, Sun AQ, Fan YB, Deng XY. Comparative study of Newtonian and non-Newtonian simulations of drug transport in a model drug-eluting stent. Biorheology. 2012;49(4):249-259.

19. Kandail H, Hamady M, Xu XY. Patient-specific analysis of displacement forces acting on fenestrated stent grafts for endovascular aneurysm repair. J Biomech. 2014;47(14):3546-3554.

20. Massai D, Soloperto G, Gallo D, Xu XY, Morbiducci U. Shear-induced platelet activation and its relationship with blood flow topology in a numerical model of stenosed carotid bifurcation. European Journal of Mechanics B-Fluids. 2012;35:92-101.

21. Del Alamo JC, Meili R, Alonso-Latorre B, Rodriguez-Rodriguez J, Aliseda A, 
Firtel RA, et al. Spatio-temporal analysis of eukaryotic cell motility by improved force cytometry. Proc Natl Acad Sci U S A. 2007;104(33):1334313348.

22. He $\mathrm{X}, \mathrm{Ku} \mathrm{DN}$. Pulsatile flow in the human left coronary artery bifurcation: average conditions. J Biomech Eng. 1996;118(1):74-82.

23. Himburg HA, Grzybowski DM, Hazel AL, LaMack JA, Li XM, Friedman MH. Spatial comparison between wall shear stress measures and porcine arterial endothelial permeability. Am J Physiol Heart Circ Physiol. 2004;286(5):H19161922.

24. Lee SW, Antiga L, Steinman DA. Correlations among indicators of disturbed flow at the normal carotid bifurcation. J Biomech Eng. 2009;131(6):061013.

25. Verhoeven EL, Katsargyris A, Bekkema F, Oikonomou K, Zeebregts CJ, Ritter W, et al. Editor's Choice - Ten-year Experience with Endovascular Repair of Thoracoabdominal Aortic Aneurysms: Results from 166 Consecutive Patients. Eur J Vasc Endovasc Surg. 2015;49(5):524-531.

26. Oderich GS, Ribeiro M, Hofer J, Wigham J, Cha S, Chini J, et al. Prospective, nonrandomized study to evaluate endovascular repair of pararenal and thoracoabdominal aortic aneurysms using fenestrated-branched endografts based on supraceliac sealing zones. J Vasc Surg. 2017;65(5):1249-1259 e1210.

27. Motta F, Crowner JR, Kalbaugh CA, Marston WA, Pascarella L, McGinigle KL, et al. Outcomes and complications after fenestrated-branched endovascular aortic repair. J Vasc Surg. 2019;70(1):15-22. 
28. Georgiadis GS, van Herwaarden JA, Antoniou GA, Hazenberg CE, Giannoukas AD, Lazarides MK, et al. Systematic Review of Off-the-Shelf or PhysicianModified Fenestrated and Branched Endografts. $J$ Endovasc Ther. 2016;23(1):98-109.

29. Premprabha D, Sobel J, Pua C, Chong K, Reilly LM, Chuter TA, et al. Visceral branch occlusion following aneurysm repair using multibranched thoracoabdominal stent-grafts. J Endovasc Ther. 2014;21(6):783-790.

30. Xue Y, Liu X, Sun AQ, Zhang P, Fan YB, Deng XY. Hemodynamic Performance of a New Punched Stent Strut: A Numerical Study. Artif Organs. 2016;40(7):669-677.

31. Reininger AJ, Heijnen HF, Schumann H, Specht HM, Schramm W, Ruggeri ZM. Mechanism of platelet adhesion to von Willebrand factor and microparticle formation under high shear stress. Blood. 2006;107(9):3537-3545.

32. Kolandaivelu K, Swaminathan R, Gibson WJ, Kolachalama VB, NguyenEhrenreich KL, Giddings VL, et al. Stent thrombogenicity early in high-risk interventional settings is driven by stent design and deployment and protected by polymer-drug coatings. Circulation. 2011;123(13):1400-1409.

33. Suess T, Anderson J, Danielson L, Pohlson K, Remund T, Blears E, et al. Examination of near-wall hemodynamic parameters in the renal bridging stent of various stent graft configurations for repairing visceral branched aortic aneurysms. J Vasc Surg. 2016;64(3):788-796.

34. Srinivasan M, Prasad A. Metal fatigue in myocardial bridges: stent fracture 
limits the efficacy of drug-eluting stents. J Invasive Cardiol. 2011;23(6):E150-

459

460

461

462

463

464

465

466

467

468

469

470

471

472

473

474

475

476

477

478

479 152.

35. Karino T. Microscopic structure of disturbed flows in the arterial and venous systems, and its implication in the localization of vascular diseases. Int Angiol. $1986 ; 5(4): 297-313$

36. Sun AQ, Fan YB, Deng XY. Numerical comparative study on the hemodynamic performance of a new helical graft with noncircular cross section and SwirlGraft. Artif Organs. 2010;34(1):22-27.

37. Walker J, Kaushik S, Hoffman M, Gasper W, Hiramoto J, Reilly L, et al. Longterm durability of multibranched endovascular repair of thoracoabdominal and pararenal aortic aneurysms. J Vasc Surg. 2019;69(2):341-347.

38. Ou J, Tang AYS, Chiu TL, Chow KW, Chan YC, Cheng SWK. Haemodynamic Variations of Flow to Renal Arteries in Custom-Made and Pivot Branch Fenestrated Endografting. Eur J Vasc Endovasc Surg. 2017;53(1):133-139.

39. Avrahami I, Brand $\mathrm{M}$, Meirson $\mathrm{T}$, Ovadia-Blechman $\mathrm{Z}$, Halak M. Hemodynamic and mechanical aspects of fenestrated endografts for treatment of Abdominal Aortic Aneurysm. European Journal of Mechanics B-Fluids. 2012;35:85-91. 
Table1. Demographic characteristics and clinical outcome of the three cases.

\begin{tabular}{|c|c|c|c|}
\hline Index & Case 1 & Case 2 & Case 3 \\
\hline Height(cm) & 156 & 180 & 172 \\
\hline Weight(kg) & 61 & 90 & 79 \\
\hline BMI & 25.1 & 27.8 & 26.7 \\
\hline Gender & female & male & male \\
\hline Age (Year) & 65 & 61 & 68 \\
\hline $\begin{array}{l}\text { Follow-up time } \\
\text { (months) }\end{array}$ & 24 & 20 & 16 \\
\hline $\begin{array}{l}\text { Blood pressure } \\
(\mathrm{mmHg})\end{array}$ & $130 / 91$ & $120 / 81$ & $150 / 90$ \\
\hline BSG-IST & No & Yes & No \\
\hline Velocity $(\mathrm{m} / \mathrm{s})$ & $1.08 \pm 0.23$ & $0.40 \pm 0.17$ & $1.01 \pm 0.12$ \\
\hline TAWSS (Pa) & $0.93 \pm 0.09$ & $0.65 \pm 0.06$ & $0.88 \pm 0.06$ \\
\hline OSI & $0.06 \pm 0.03$ & $0.30 \pm 0.04$ & $0.11 \pm 0.06$ \\
\hline RRT (s) & $1.94 \pm 0.24$ & $9.27 \pm 2.61$ & $2.52 \pm 1.00$ \\
\hline Stent-graft & $\begin{array}{c}\text { Endurant,36-14- } \\
\text { 120mm,6*150mm,Vi } \\
\text { abahn, Gore }\end{array}$ & $\begin{array}{l}\text { Endurant,38-14- } \\
\text { 120mm,6*150mm, } \\
\text { Viabahn, Gore }\end{array}$ & $\begin{array}{c}\text { Endurant,30-14- } \\
\text { 120mm,6*150mm,Via } \\
\text { bahn,Gore }\end{array}$ \\
\hline
\end{tabular}
stress; OSI, oscillatory shear stress index; RRT, relative residence time. 
489

490

491

492

493

494

495

496

497

498

499

500

501

502

503

504

505

506

507

508

509

510

511

Figure 1. CT images and modeling images. (a) The CT angiogram (pre- and postoperative) of the three cases, the pre-operative CT angiogram of three cases are shown in the upper line, and the post-operative CT angiogram of three cases are shown in the lower line. (b) Meshes of three models and the flow split ratio of every single branch vessel of three cases. (c) Inlet velocity waveform of the aorta (four typical moments are marked in a cycle, $\mathrm{t}_{1}=0.125 \mathrm{~s}, \mathrm{t}_{2}=0.225 \mathrm{~s}$ (the peak systolic moment), $\mathrm{t}_{3}=0.435 \mathrm{~s}$ (the end systolic moment), $\left.\mathrm{t}_{4}=0.62 \mathrm{~s}\right)$.

Figure 2. The representative illustration of the construction of the physician-modified branched stent-grafts and the process of the stent-graft implantation. (a) split a Gore Viabahn ${ }^{\mathrm{TM}}$ into 3 sections. (b) sewing the caudally-directed cuff to a Medtronic Endurant ${ }^{\mathrm{TM}}$ bifurcated stent-graft. (c) completion of the modification. (d) top view of the visceral cuff showing no gutters. (e) the operation is performed using right femoral and left brachial access. (f) a Valiant Captivia ${ }^{\mathrm{TM}}$ thoracic stent-graft is firstly deployed at descending aorta. (g) implantation the main body of the modified Endurant bifurcated stent-graft through right femoral approach and cannulating the celiac arteries preferentially through left axillary artery accesses using a long sheath and an extra-stiff guidewire. (h) sequential bridging the visceral arteries with Viabahn while reinforcement for bilateral renal arteries using self-expandable bare metal stent and dilated from the target vessel to the cuff. (i) the distal iliac limbs extension is docked and flow is restored to the lower limbs (Department of Vascular Surgery, Peking University People's Hospital. All rights reserved). 
512 Figure 3. The follow-up CT scans of three cases. Case 1 with no thrombus in the

513 branches, Case 2 with the branched stent-graft thrombosis marked with green, Case 3

514 with no thrombus in the branches.

515

516 Figure 4. Velocity streamlines and continuous contours of velocity through a cardiac

517 circle of the three cases. (a), (c) and (e) are the velocity streamlines of three cases at

518 four typical moments (t1-t4) in a cardiac cycle, (b), (d), (f) show the locations of

519 sections in each model and contours of flow velocity in marked sections at four typical

520 moments (t1-t4) in a cardiac cycle of three cases.

521

522 Figure 5. The wall shear stress (WSS) distributions of the stent-grafts in three cases.

523 The same four moments $\left(\mathrm{t}_{1}-\mathrm{t}_{4}\right)$ are selected to present the WSS in each case.

524

525 Figure 6. The time-averaged wall shear stress (TAWSS), oscillatory shear index (OSI),

526 relative residence time (RRT) distributions of the three cases. (a) TAWSS distributions.

527 (b) OSI distributions. (c) RRT distributions.

528

529 Figure 7. Statistical analysis of the four hemodynamic parameters

530 TAWSS indicates, the time-averaged wall shear stress; OSI, oscillatory shear index;

531 RRT relative residence time; NS, none significant; * indicates $\mathrm{P}<0.05$; **, $\mathrm{P}<0.001$.

532 The units of measure were standardized to the international system of unit. 


\section{Figures}
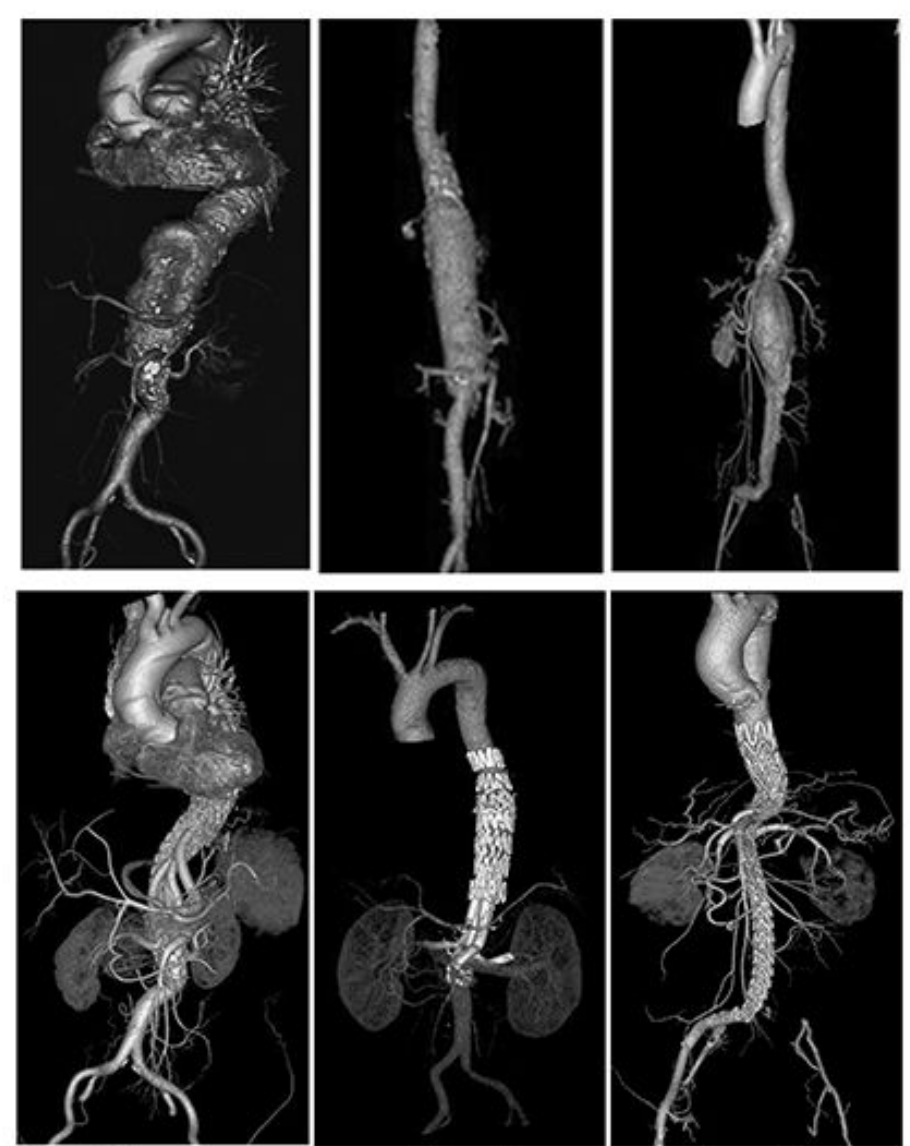

Case 1

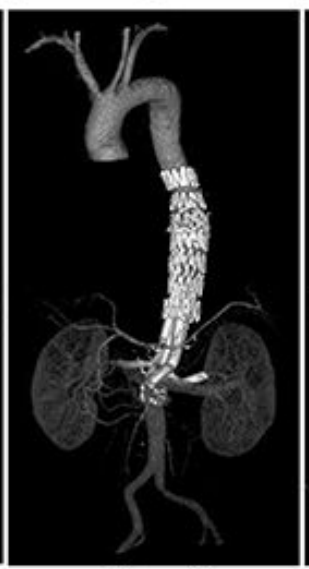

Case 2

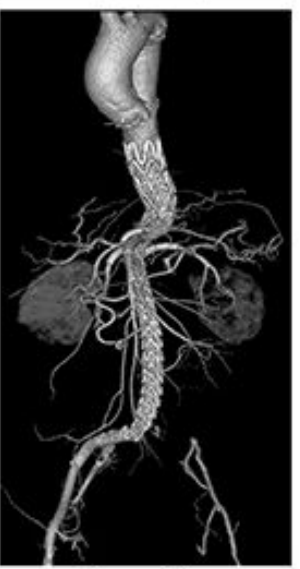

Case 3

(a)

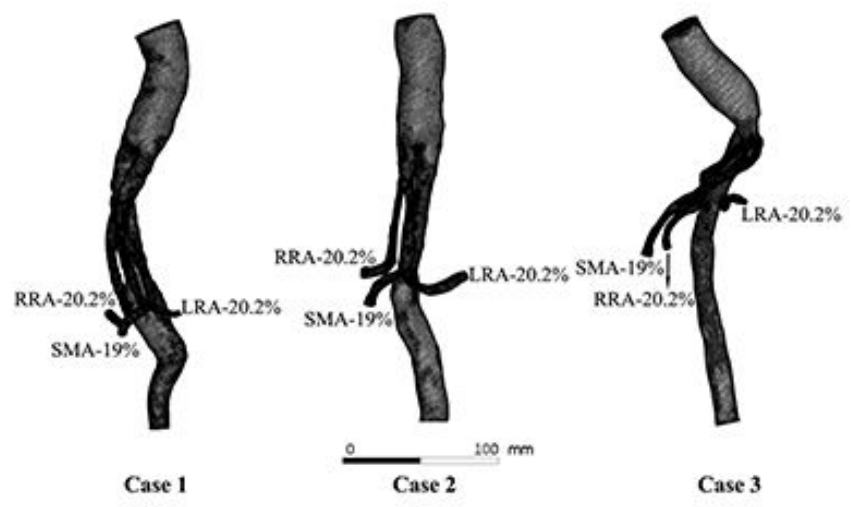

(b)

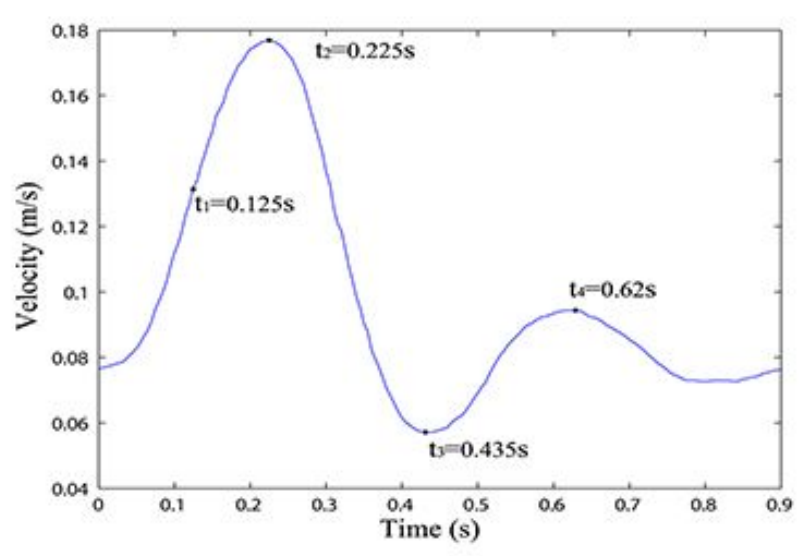

(c)

\section{Figure 1}

CT images and modeling images. (a) The CT angiogram (pre- and post-operative) of the three cases, the pre-operative CT angiogram of three cases are shown in the upper line, and the post-operative CT angiogram of three cases are shown in the lower line. (b) Meshes of three models and the flow split ratio of every single branch vessel of three cases. (c) Inlet velocity waveform of the aorta (four typical moments are marked in a cycle, $\mathrm{t} 1=0.125 \mathrm{~s}, \mathrm{t} 2=0.225 \mathrm{~s}$ (the peak systolic moment), $\mathrm{t} 3=0.435 \mathrm{~s}$ (the end systolic moment), $\mathrm{t} 4=0.62 \mathrm{~s})$. 


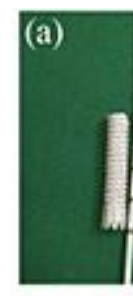

(e)

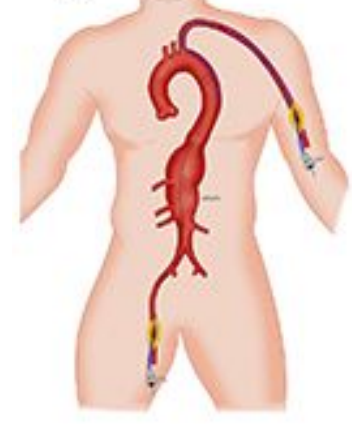

(f)

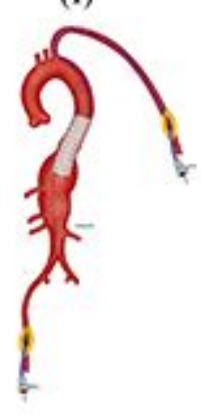

(g)

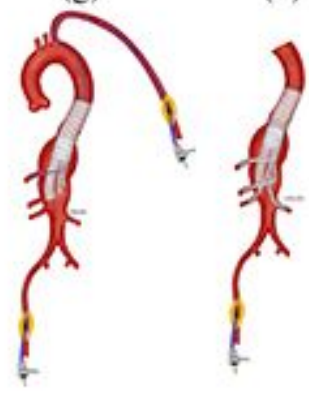

(i)

Figure 2

The representative illustration of the construction of the physician-modified branched stent-grafts and the process of the stent-graft implantation. (a) split a Gore ViabahnTM into 3 sections. (b) sewing the caudally-directed cuff to a Medtronic EndurantTM bifurcated stent-graft. (c) completion of the modification. (d) top view of the visceral cuff showing no gutters. (e) the operation is performed using right femoral and left brachial access. (f) a Valiant CaptiviaTM thoracic stent-graft is firstly deployed at descending aorta. (g) implantation the main body of the modified Endurant bifurcated stent-graft through right femoral approach and cannulating the celiac arteries preferentially through left axillary artery accesses using a long sheath and an extra-stiff guidewire. (h) sequential bridging the visceral arteries with Viabahn while reinforcement for bilateral renal arteries using self-expandable bare metal stent and dilated from the target vessel to the cuff. (i) the distal iliac limbs extension is docked and flow is restored to the lower limbs (Department of Vascular Surgery, Peking University People's Hospital. All rights reserved). 

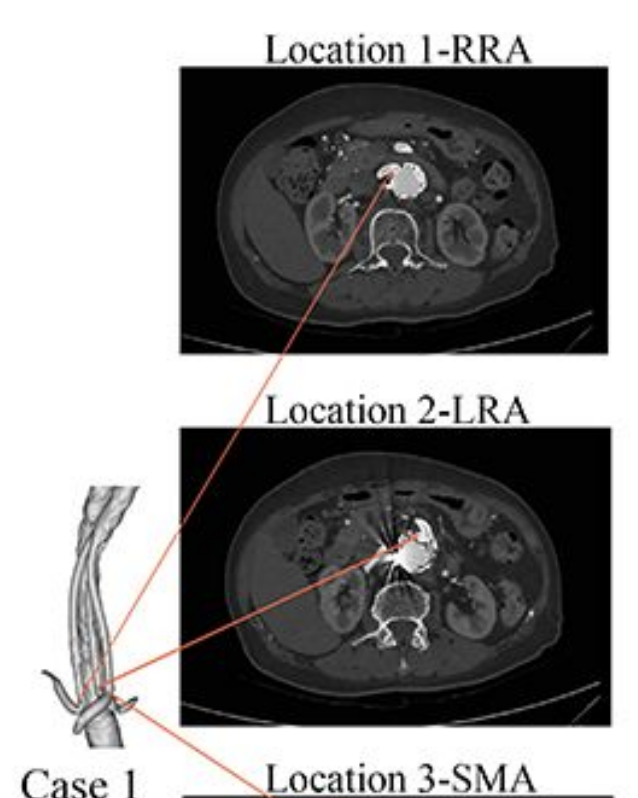

Case 1

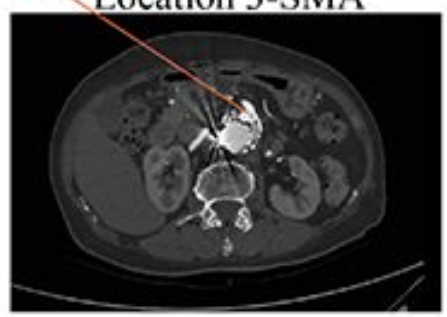

Location 1-SMA
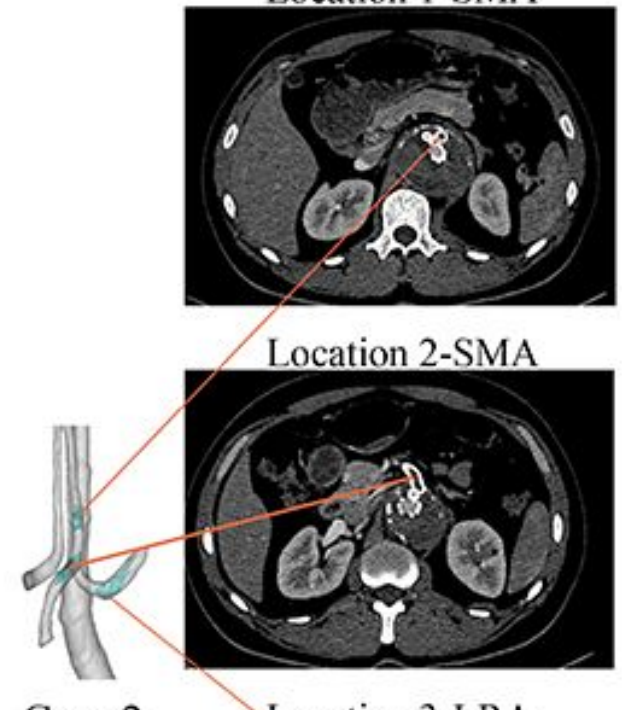

Case 2
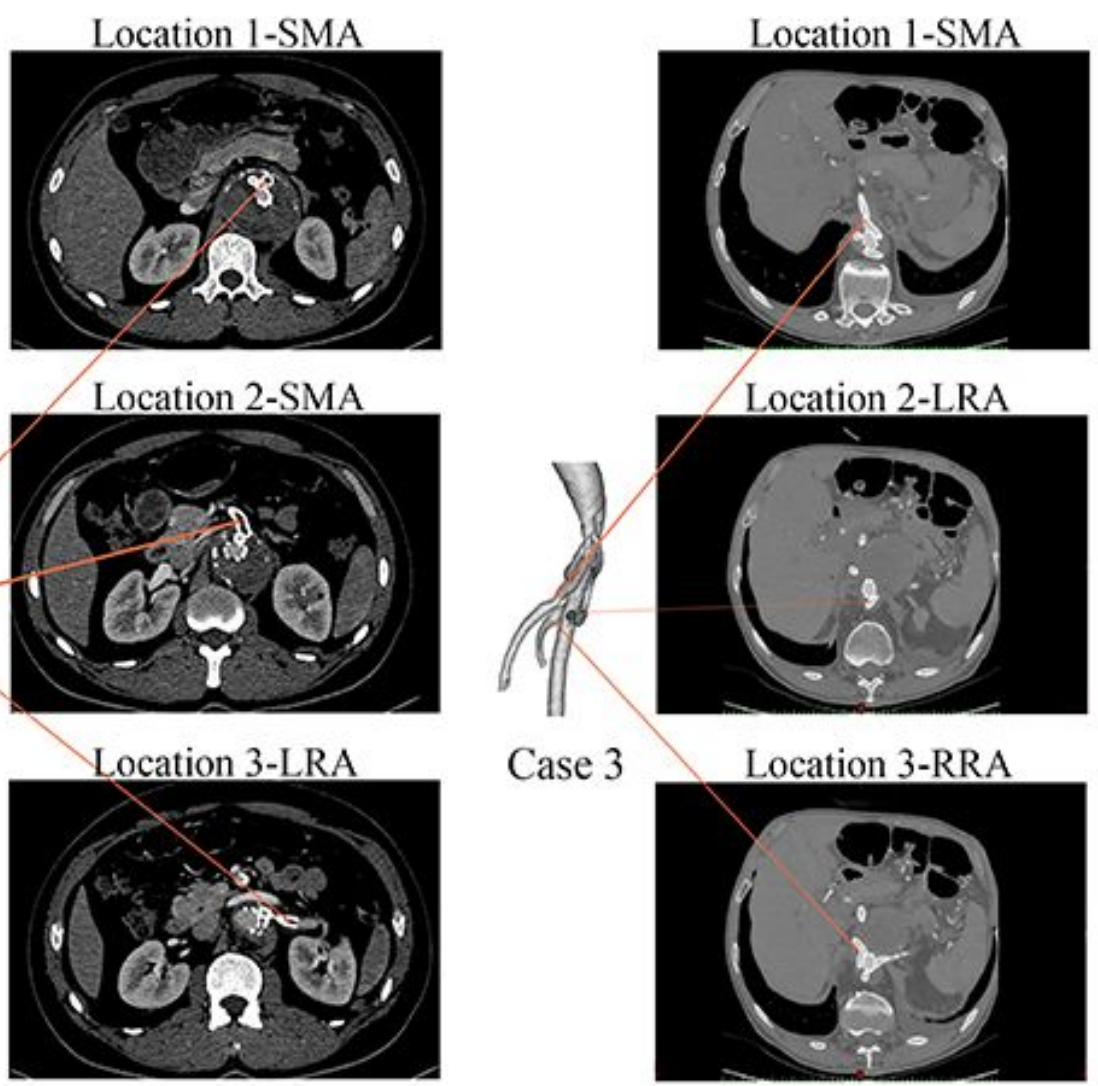

\section{Figure 3}

The follow-up CT scans of three cases. Case 1 with no thrombus in the branches, Case 2 with the branched stent-graft thrombosis marked with green, Case 3 with no thrombus in the branches. 

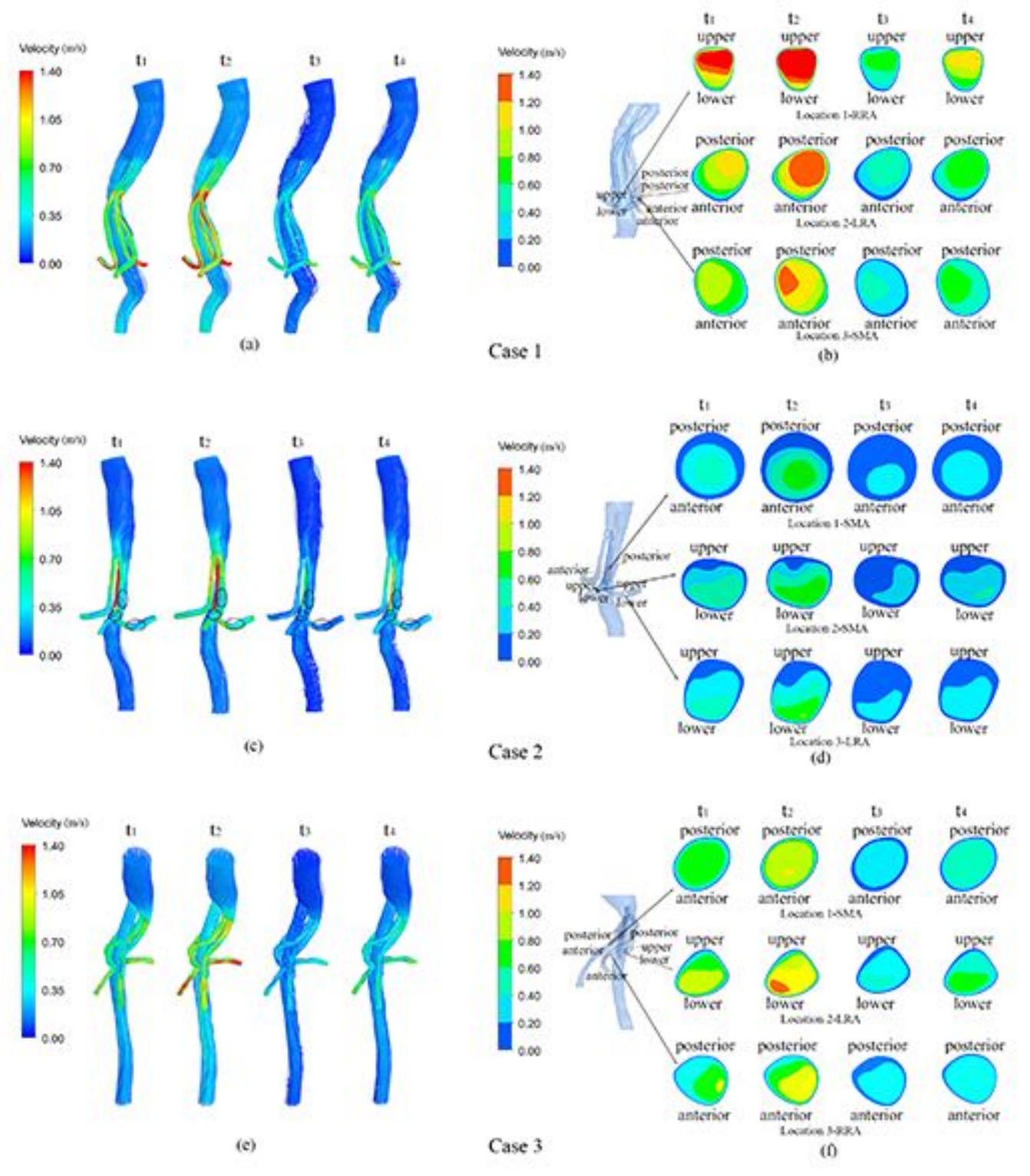

\section{Figure 4}

Velocity streamlines and continuous contours of velocity through a cardiac circle of the three cases. (a), (c) and (e) are the velocity streamlines of three cases at four typical moments (t1-t4) in a cardiac cycle, (b), (d), (f) show the locations of sections in each model and contours of flow velocity in marked sections at four typical moments (t1-t4) in a cardiac cycle of three cases. 

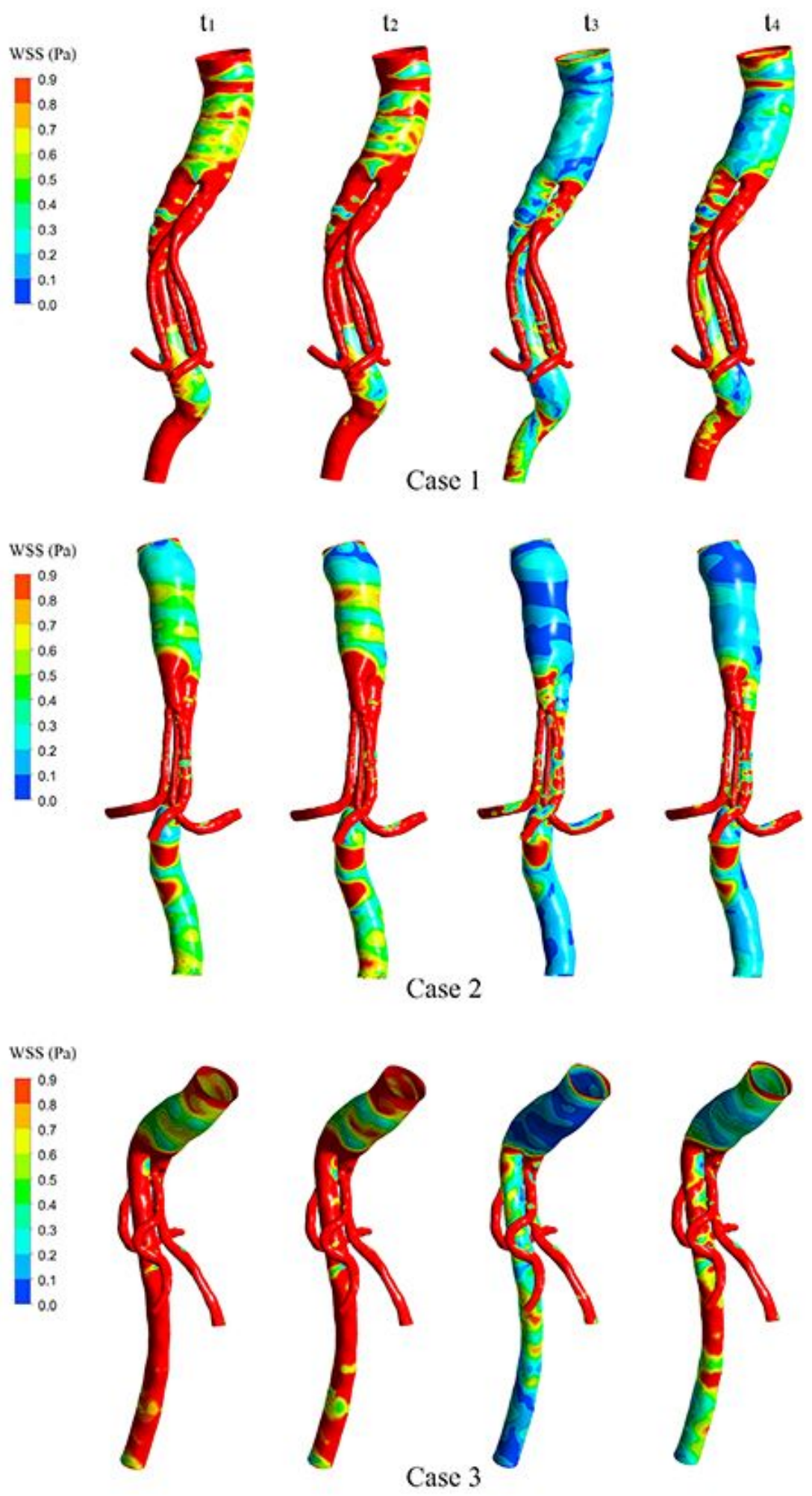

Figure 5

The wall shear stress (WSS) distributions of the stent-grafts in three cases. The same four moments (t1t4) are selected to present the WSS in each case. 

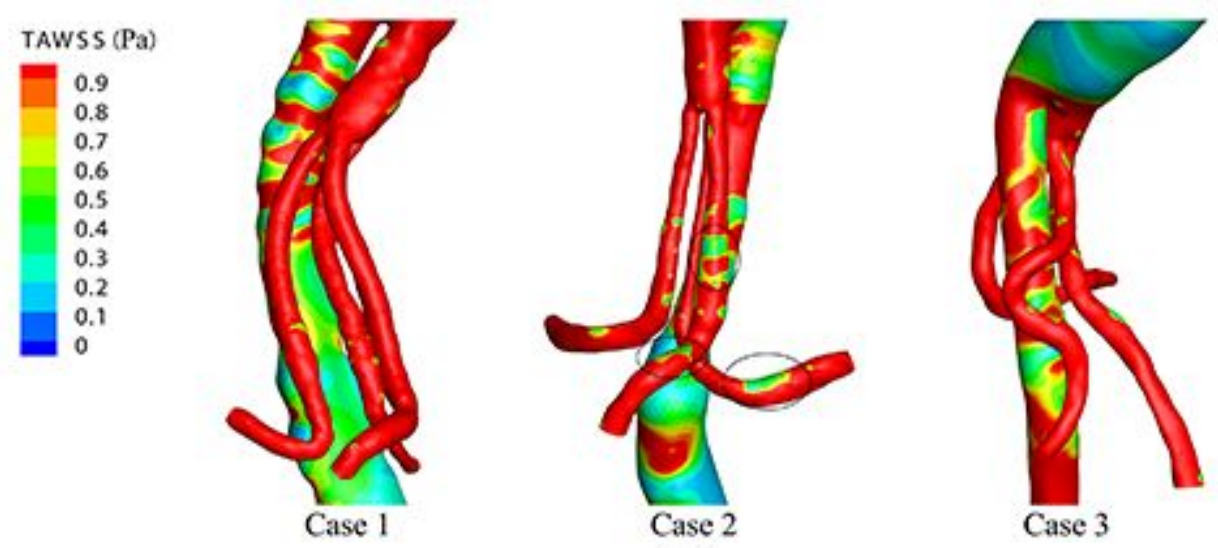

OSI
0.4
0.35
0.3
0.25
0.2
0.15
0.1
0.05
0

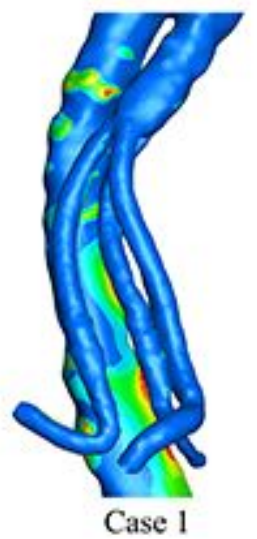

(a)

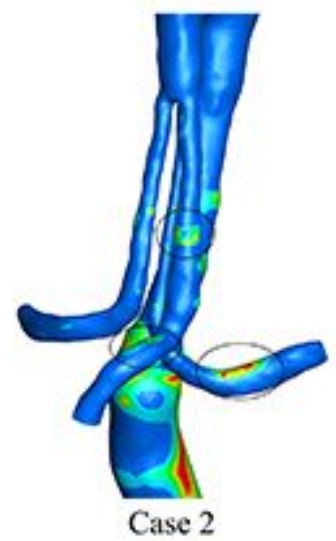

(b)
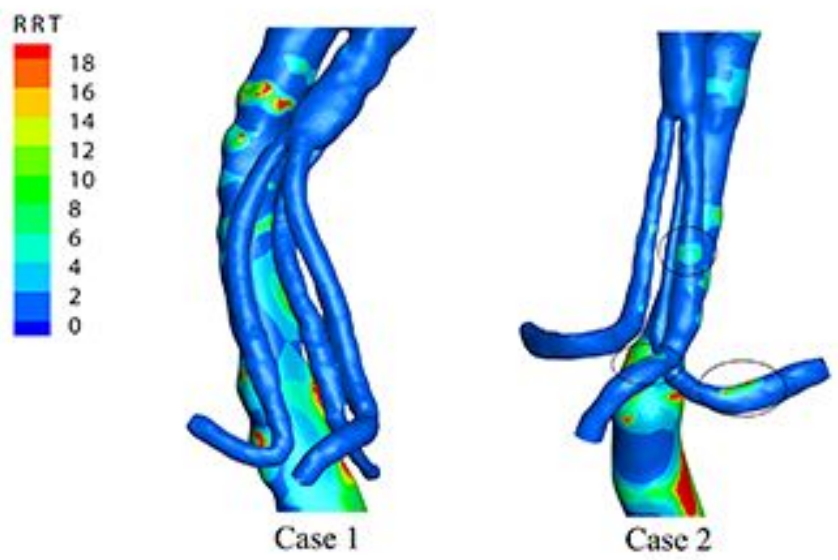

(c)
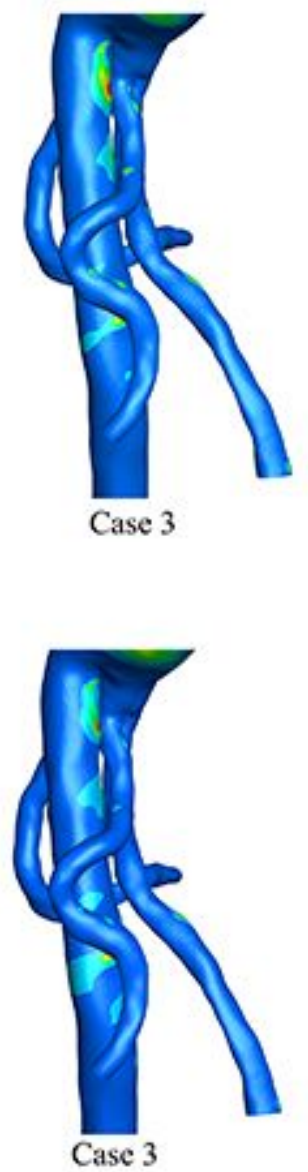

\section{Figure 6}

The time-averaged wall shear stress (TAWSS), oscillatory shear index (OSI), relative residence time (RRT) distributions of the three cases. (a) TAWSS distributions. (b) OSI distributions. (c) RRT distributions. 
Velocity

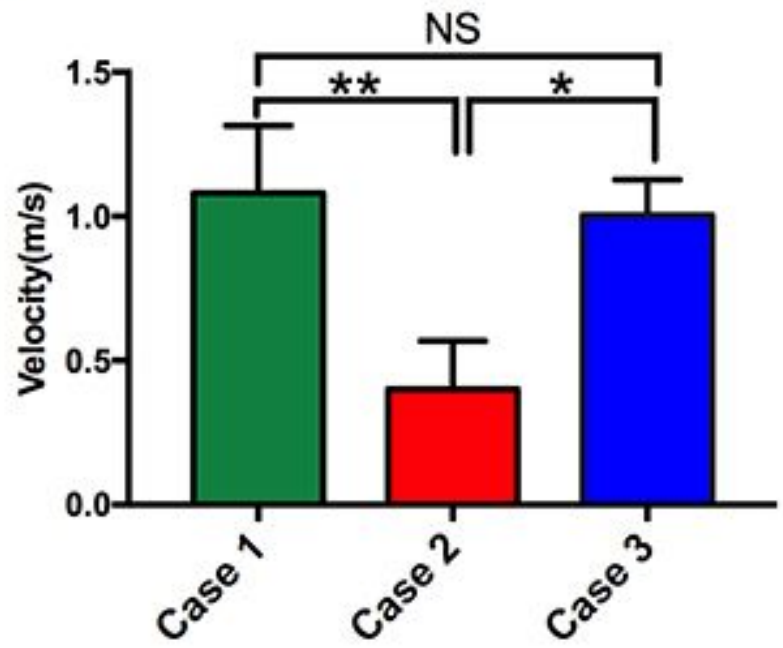

(b)

TAWSS

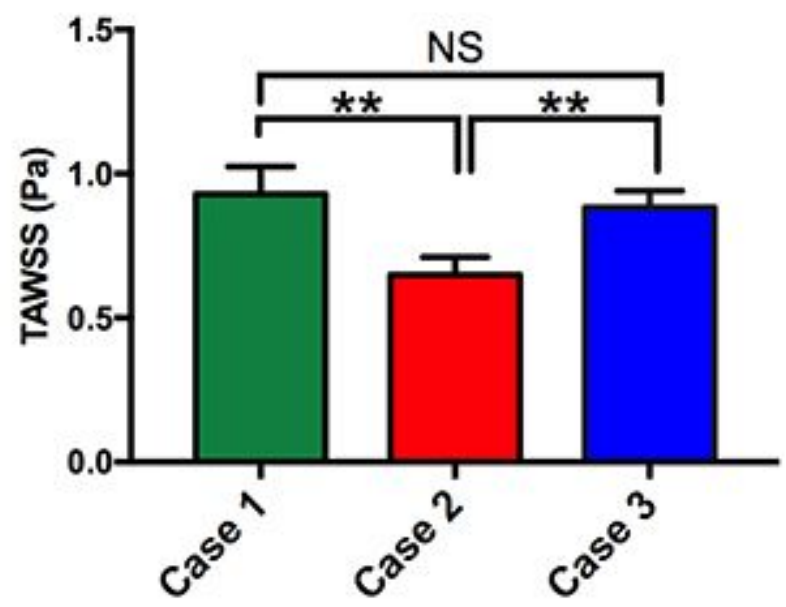

(d)

RRT

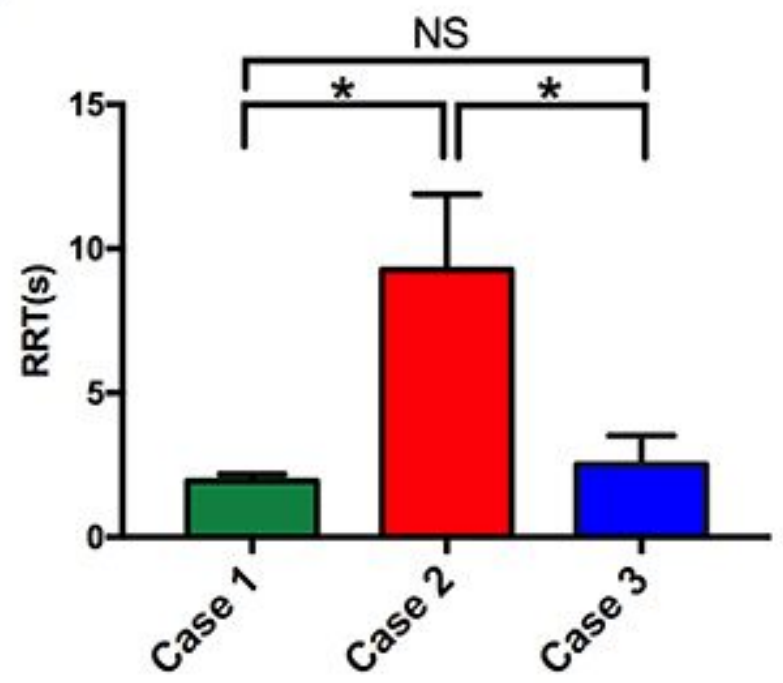

Figure 7

Statistical analysis of the four hemodynamic parameters TAWSS indicates, the time-averaged wall shear stress; OSI, oscillatory shear index; RRT relative residence time; NS, none significant; * indicates $\mathrm{P}<0.05$; $\star \star, P<0.001$. The units of measure were standardized to the international system of unit. 